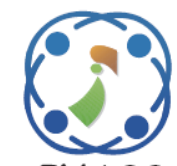

\title{
Composite Metric Based Energy Efficient Routing Protocol for Internet of Things
}

\author{
Sankar Sennan $^{1 *} \quad$ Srinivasan Palanisamy ${ }^{1}$ \\ ${ }^{l}$ Vellore Institute of Technology University, Vellore,Tamilnadu, India \\ *Corresponding author's Email: sankar.s@vit.ac.in
}

\begin{abstract}
Nowadays, there is a drastic increase in the number of sensors enabled devices connected to the Internet. Low power and lossy network (LLN) is a network, which consists of several embedded devices with limited memory, power and processing resources. Energy preservation and network lifetime enhancement are the major challenges in LNN. Hence, the routing plays a vital role in LLN, as an Internet of Things (IoT) device need to take the decision accordingly. In LNN, the inefficient route selection results in increased energy depletion, traffic and packet loss ratio. The proposed work introduces Load and battery discharge index (BDI) based composite routing metric in IPV6 Routing Protocol for Low power and lossy network (RPL). The COOJA simulator used to evaluate the performance of the proposed work. It provides the better result in terms of network lifetime and packet delivery ratio (PDR) against the routing metric such as hop count, ETX, RER, RER (BDI) and traffic load.
\end{abstract}

Keywords: Internet of things, Low power and lossy network, Energy efficiency, Load.

\section{Introduction}

The Internet of Things (IoT) is a collection of interrelated physical devices connected to the internet, that able exchange information between them without human involvement [1-2]. The low power and lossy network (LLN) is a network that consists of few dozen to thousands of routers and sensor enabled physical devices [3-5]. However, the interconnecting links are instability and high loss rates in LLN. In order to overcome those limitations, IETF evaluated and identified the incapability of all the conventional protocols such as IP based routing protocols for wireless sensor networks (WSN), adhoc on-demand distance vector(AODV), optimized link state routing(OLSR), open shortest path first (OSPF), intermediate system to intermediate system (IS-IS) for ad-hoc network and Optimal Secured Energy Aware Protocol [6-8]. Finally, IETF-ROLL working group has standardized the RPL protocol and it has the capability of adopting and fulfils the requirement of LLN. RPL follows the multi hop. Transmission of data packet from source to root node. The optimal route selection in a way to utilize energy and improves the quality of service. It supports the point-to-point, Point -to-multipoint and multipoint-to- point data traffic [9].

The proposed work primary concern is to improve the energy preservation and network lifetime enhancement. Whenever the participant node wishes to join in Destination Oriented Directed Acyclic Graph (DODAG), it always keeps in mind of the following factors, such as traffic load, battery discharge, link quality and loop freeness, etc. The proposed work introduces Load, and battery discharge index (BDI) based composite routing metric in RPL. Periodically, DODAG sends the DODAG Information Object (DIO) messages to all participant nodes. The participant node chooses the best parent from DODAG rank. The rank calculates from minimum load and BDI values of the node. Finally, the participant node selects the best parent from DODAG. Thus, it reduces the multi point-topoint data traffic in upward routing, avoid the nodes creating the hole in the network due to battery depletion and decrease the packet loss ratio. 
The paper is organized as follows: Section 2 describes the related work. Section 3 describes the proposed routing metric. Section 4 represents the result and analysis and final section is the conclusion and future work.

\section{Related work}

In this section briefly discuss about RPL routing protocol and its metrics based routing issues.

\subsection{Rpl}

RPL is a proactive distance vector source routing protocol, specially designed for LLN. It contains the structure as Destination Oriented Directed Acyclic Graph (DODAG). It follows tree topology and each node wants to reach the single destination. The top of the node called root. The upward route directs towards the root, and downward route directs away from the root. At a time, RPL protocol generates more than one DODAG in a network, and each DODAG considers an instance of RPL. The entire instance maintains separate RPL instance ID or DODAG ID. Each node has own IP address.

The root node acts as a border router, and remaining nodes act as child or host node in LLN. The host node gets the address from root ID prefixes. The root node or sink node maintains the entire routing table about the network. The root node always keeps the storing mode, and it gives the privileges to some child node, which also maintains the routing information in the part of the network. In storing mode, the node can collect and forward the data to another node. However, the non-storing mode, the node can only forward the data to another node. RPL protocol has four control messages such as DODAG Information Solicitation (DIS), DODAG Information Object (DIO), DODAG Advertisement Object (DAO), DODAG Advertisement Object- Acknowledgment (DAOACK).

Initially, DODAG contains the root node or border router. The participant node wants to join with existing DODAG, and it needs to send the DIS control message to DODAG. DODAG multicasts the DIO control messages to all participant nodes. It contains the objective function and rank details. DODAG enables the trickle timer. The DODAG wait to get the DAO control messages from participant node. Within the trickle time, the participant node will send the DAO control messages to DODAG, and it accepts the participant request and sends the DAO-ACK control messages to participant's node. The rank calculates from objective function. The objective function provides the optimal solution for select the best parent and sends the data towards the root [9-10].

\subsection{Primary routing metric}

The primary routing metrics based on the node and link behaviour of the DODAG. Link based primary routing metrics are ETX, link quality level (LQL), Latency and Packet Loss [9]. Node based primary routing metrics are Hop count, Residual energy and RSSI. Many RPL protocol implementations followed by the routing metrics such as Hop count and expected transmission count (ETX) [11]. Using Hop count as a primary routing metric, which minimize the number of node present in the route and minimize the node depletion energy. This metric limitation is, whenever the node energy exhausted present in the network and it required to retransmit the data again [5].

Muneer bani yassein et'al proposed energy aware objective function for IoT routing protocol. The participant node selects the parent node based on the less power consumption of the node. Thus, the objective function balanced power consumption among the nodes. It ensured to improve the packet reception ratio and network lifetime [12].

$\mathrm{Gen} \mathrm{Xu}$ and Gang Lu proposed multipath routing protocol for directed acyclic graph (DAG) based wireless sensor networks with mobile sink (MDMR). It considered the metrics such as hop count, node energy and link quality indication. The MDMR protocol constructed the DAG and provided the redundant path. Whenever the node occur the link failure, it chose the alternate path to send the data [13].

Oana Lova et'al proposed the expected lifetime metric using multipath approach. RPL exploited the DAG topology and the participant node sent the data packet probabilistically forward the traffic in to several nodes. Finally, it improved the network lifetime and routing reliability [14].

P.Di Marco et'al proposed MAC aware routing metrics for the IoT. It focused to analyze the MAC and routing protocols for IoT. The existing routing metric unable to integrate between MAC and RPL protocol. It introduced a novel metric $\mathrm{R}$ metric and $\mathrm{Q}$ metric based on joint MAC and routing model. It provided the better performance than existing routing metric [15].

Expected Transmission Count (ETX) is a link metric, which minimize expected total number of data packet transmission and including retransmission. ETX incorporated the result of link 
loss ratio, asymmetry in the loss ratio between the two direction of each link and interference among the link path [16].

Mamoun Qasem et'al proposed a load balancing objective function for IoT routing. In IPv6, two objective functions are already standardized namely objective function zero (OF-0) and objective function (MRHOF). However, this objective function balanced the data traffic across the network nodes. The participant node wants to select the parent node, which based on the number of child count present over the path. It introduced the modified DIO message format, which reduces the control overhead among the network [17].

\subsection{Composite routing metric}

The composite routing metrics involves combination of more than one metric of node and link behaviour of the DODAG. The composite routing metric solution fulfils the requirement of convergence, optimality, and loop-freeness. Many researchers involved and contributed to improve the efficiency of routing in LLN.

Juan Pablo Yunis et'al proposed an energy efficient routing protocol using composite metrics. Hop count and remaining energy based composite metric used as objective function. It analyzed the performance latency, energy consumption and packet loss in different scenario [18].

Lai Nguyen et'al proposed a composite metric based dynamic routing protocol in LLN. A LLN routing protocol is an application specific protocol. In general, it followed the weighted composite primary metric (ETX, Hop count, Load, through put etc) that achieved convergence, path control and loop free properties. This work pointed out the relation between network performance and routing metric criteria [19].

Ali Hassan et'al proposed an energy efficient composite metrics based RPL protocol in LLN. It based on residual energy (RER), battery discharge index (BDI) and expected transmission count (ETX). These works improved the network lifetime and reduced the battery depletion. However, it didn't give preferences for link quality (ETX) [5].

Patrick Olivier kamgueu et'al proposed on fuzzy logic based routing metric in low power and lossy networks. The key objective of design an efficient sensor network protocol used to minimize the energy consumption and extended the network lifetime. This work is taken into account the metrics are ETX, delay, energy. It proposed fuzzy logic based composite routing metric and provided the quality of service and improved the energy efficiency [20].

Olfa Gaddour et'al proposed fuzzy logic based composite routing metric for the RPL routing protocol. It used the metrics are End-to-End delay, Hop count, Residual energy and ETX. This work proposed fuzzy logic based objective function, which considered the above-mentioned metrics. It provided the quality of service in different kind of application [21].

Silvia Capone et'al proposed an energy efficient and reliable composite metric for RPL. This work considered both link and node metric. The metrics are residual energy and ETX. First, it concentrated on energy balancing among the nodes using residual energy. Second, it concentrated on link reliability using ETX. It deeply investigated the issues of reliability and energy efficiency in LNN [22].

\section{Proposed routing metric}

In IoT, Routing protocols for low power and lossy network (LLN) are facing the challenges like lack of infrastructure, unreliable wireless links and constrained resources. The network lifetime decreases due to multi hop routing, traffic overhead. To avoid those issues, the proposed work introduces the composite routing metric, and it improves the network lifetime and uses the energy efficient way. It considers the path to build a route. The path Px $=\mathrm{N} 1 \oplus \mathrm{N} 2 \oplus \cdots \oplus \mathrm{Nn}$ where Px $\in \Phi$ and N1, N2, $\cdots, \mathrm{Nn}$ are traversed nodes. Each node associated with battery discharge index (BDI) and Load in the network.

\subsection{Load}

Network data traffic is an amount of data transfer across the network at given amount of time. Mamoun Qasem et'al (2017) introduced a Load balancing technique, and it is standardized by IETF. The load is a metric, and it uses to balance the data traffic. Load calculates based on the number of children present in the parent node [17]. The DODAG node broadcasts DIO messages to all participant nodes. The sender node or participant node calculates the children count for each preferred parent node. Finally, the DODAG generates the rank based on the cumulative children count present in the path. The participant node chooses the parent from preferred parent list and the selection based on the Load. The metric Load follows the Additive property, and the traffic load calculation is given in Eq. (1) and Eq. (2). 


\subsubsection{To calculate load}

To calculate the load of Path (x) based on cumulative of node traffic or child set.

$$
\operatorname{Load}(P x)=\sum_{N=1}^{n} N o d e_{-} \operatorname{Traffic}(N)
$$

To calculate the node traffic based on the cumulative of children count.

$$
\text { Node_Traffic }(N)=\sum_{i=1}^{n} \text { children_count }(i)
$$

The load-balancing algorithm is represented in below.

\section{Load Balancing Algorithm}

1: Input:

2: ReceivedDIO [InstantID, Version Number, Rank, ParentID]

3: ReceivedDAO [InstantID, Version Number, RankID]

\section{4: begin}

5: $\quad$ ParentNode $\leftarrow$ DIO;

6: $\quad$ senderNode $\leftarrow \mathrm{DAO}$;

7: While ParentNodeID=SenderNode_Prefered_ ParentID do

8: Childeren_Set++;

9: end

10: While SenderNode checks the Cumulative children Count of Prefered_ParentNode do

11: if is it least cumulative children Count then

12: $\quad$ Sender Node added in DODAG

13: else

14: $\quad$ Sender Node discards the preferred parent

15: end

$$
\text { Node }
$$

16: $\quad$ Broadcast updated DIO.

\subsection{Battery discharge index (BDI)}

The battery discharge index derives from residual energy. The residual energy calculates from each node of RER (Ni), i.e., initial energy and current energy. The residual energy calculation formula is given in Eq. (3).

$$
R E R(N i)=\frac{\text { Ecurrent }}{\text { Einitial }}
$$

The Battery discharge Index calculates for node
"N $\mathrm{N}_{\mathrm{i}}$ " as (1- Residual Energy).The BDI $\left(\mathrm{N}_{\mathrm{i}}\right)$ calculation is given in Eq. (4).

$$
B D I(N i)=(1-R E R(N i))
$$

The Battery Depletion Index of Path Px calculation is given in Eq. (5). If follows the productive rule.

$$
B D I(P x)=\prod_{i=1}^{n} B D I(N i)
$$

\subsection{Objective function (OF)}

The objective function (OF) defines how to select DODAG node and optimize the route in RPL instance. The proposed composite metric based objective function is to enhance the energy efficiency. This objective function focus on following issues such as data traffic in multipointto- point communication, bottle neck occur near sink node, loop-freeness. The OF is denoted in Eq. (6), and it gives the equal preference to both the metric. It applied different weight values for this simulation and finally set the weight values $w 1=0.5$ and $\mathrm{w} 2=0.5$.

$$
\min O F_{L E}(L B, B D I)=w 1 \times \operatorname{Load}(P i)+w 2 \times B D I(P i)
$$

\subsection{Rank calculation}

The rank calculates from parent rank and rank increase. The rank increase calculates from step and MinHopRankIncrease, where MinHopRankIncrease default value is 256 [8]. The step value calculates from objective function, and it returns the scalar value. The rank calculation formula is denoted in Eq. (7) and Eq. (8).

$$
\operatorname{Rank}(N)=\operatorname{Rank}(P N)+\text { Rank increase }
$$

Rank increase $=$ Step + MinHopRankIncrease $(8)$

\subsection{Parent selection process}

The DODAG node periodically sends the DODAG Information Object (DIO) to all participant nodes. The participant node wants to send the data to another node towards root and it needs to choose the preferred parent from DODAG. The best parent selection based on the DODAG rank. The Rank calculates from parent rank and rank increase. The rank increase calculates from step value and MinHopRankIncrease default value is 256 . The step 


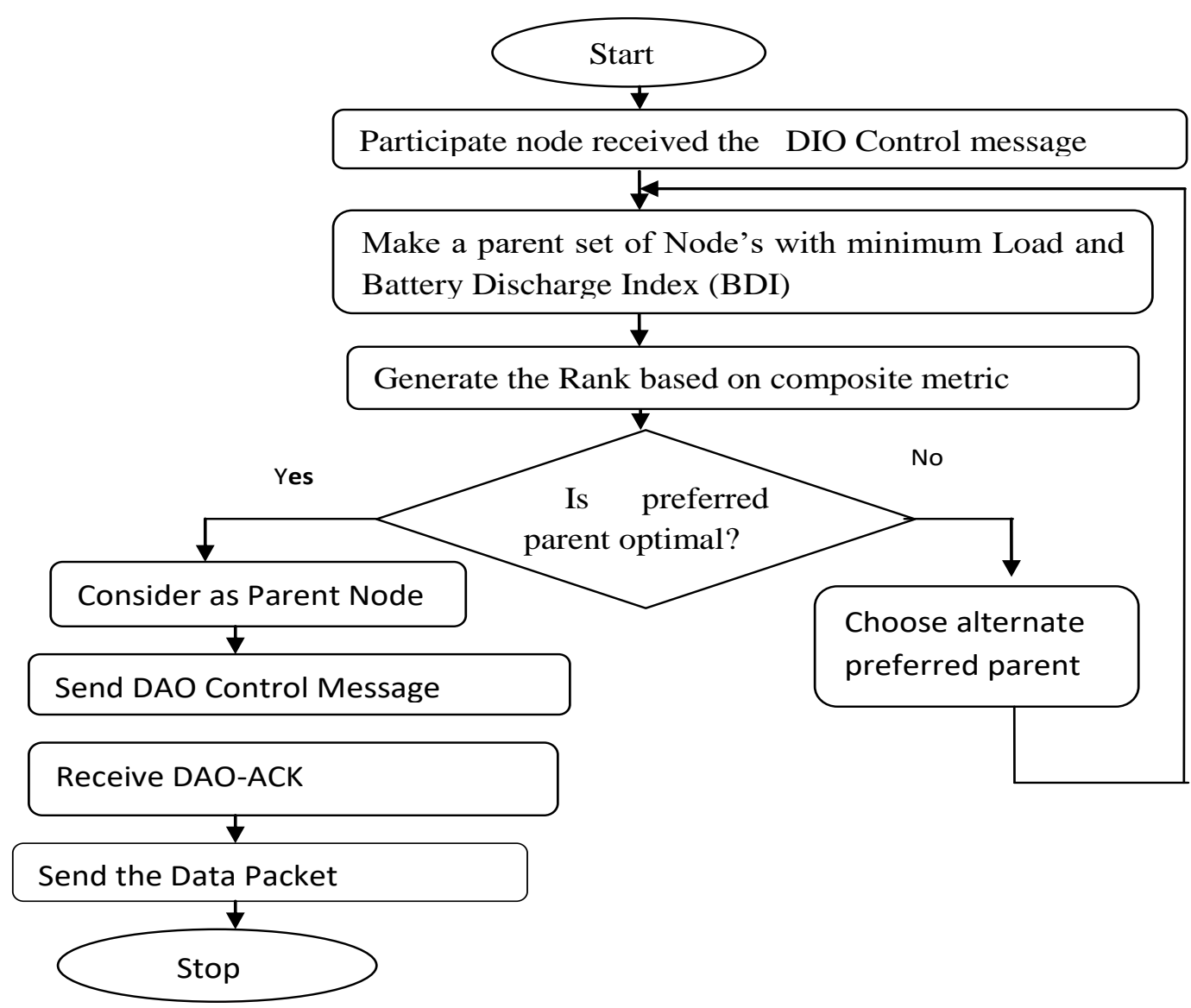

Figure.1 Parent selection and data forwarding mechanism

value calculates from objective function and it returns the scalar value.

The proposed work introduces Load, BDI based composite metric as an objective function, and it gives equal preferences to both the metrics. Load metric follows the additive property, and BDI follows the productivity property. During the parent selection, participant node prefers the minimum value of objective function. DODAG enables the trickle timer. The current interval size (I) ranges from $I_{\min }$ to $I_{\max }$. Generally, RPL protocol default values are $I_{\min }=12 \mathrm{~ms}$ and $I_{\text {doubling }}=10 \mathrm{~ms}$. Before sending the DIO message, the trickle time counter c initialize as zero and starts to send the DIO messages to neighbour nodes. DODAG expects the DODAG Advertisement Object (DAO) messages from participant node. When the trickle time expires the interval "I", the DODAG extends the interval "I" value from I min to I doubling. If again trickle timer expires and unable to receive the DAO control messages from participant nodes, then the DODAG node resets the trickle time and send the DIO control messages to all participating node. The participant node sends the DODAG Advertisement Object (DAO) to DODAG.

It sends the DODAG Advertisement ObjectAcknowledgement (DAO-ACK) to participant node.
The participant node receives the DODAG-ACK from MAC layer. The parent selection process has represented an algorithm and Workflow mentioned below.

\section{Parent Selection Algorithm}

1: Input:

Node N, ParentNodeID, Participant

2: Output:

Node_ParentID, BestParent_Rank $=\infty$;

Preferred_Parent $(\mathrm{N})$

3: for Preferred Parent $\in$ Parent _List do

4: $\operatorname{Rank}(\mathrm{N}) \leftarrow \operatorname{Rank}(\mathrm{PN})+$ Rank increase;

5: Rank increase $\leftarrow$ Step + MinHopRankIncrease;

6: Step $=w 1 \times$ LB $(\mathrm{Pi})+\mathrm{w} 2 \times$ BDI $(\mathrm{Pi})$;

7: If BestParent_Rank $>=$ Prefered_ParentRank(P) then

8: BestParent_Rank $\leftarrow$ Prefered_ParentRank (P);

9: End if

10: end

11: While Prefered_Parent $\operatorname{Rank}(\mathrm{P})=$ Best

Parent_Rank do

12: ParticipantNode_ParentID $\longleftarrow$ Preferred_Parent NodeID;

13: end 


\section{Results and analysis}

The proposed work enhanced the features of standardized RPL and evaluated the performance in terms of network lifetime, Packet Delivery Ratio. The simulation has been conducted using COOJA network simulator [23].

Contiki is an open source operating system and specifically it designed for Internet of Things. It supports IPv6, 6LoWPAN, RPL, etc., It provides the low power communication for highly resource constrained devices. The lossy nature of the wireless medium simulated using unit disk graph model available in COOJA simulator.

In this simulation, RPL protocol supports the multipoint-to-point traffic from sender node to DODAG root. The simulation parameters has given in Table-1 and the default radio duty cycling mechanism used in MAC layer [24].To analyze the performance of proposed work against existing objective function such as Hop count, ETX, RER,

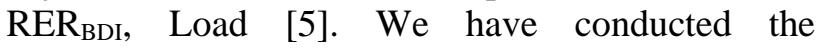
experiment and manually fine-tune the weight values of routing metric for getting more efficiency. The proposed work provided the better performance against Hop count, ETX, RER, RER BDI $_{\text {and }}$ Load where the weight values $w 1$ and $\mathrm{w} 2$ are 0.5 .

The performance of the proposed work is assessed by Packet delivery ratio and network lifetime. The details of network lifetime and packet delivery ratio are discussed in below.

\subsection{Network lifetime}

Network lifetime is a prominent criterion for evaluating the efficiency of routing protocol for resource-constrained devices [25]. The simulation has been conducted and number of nodes is taken into account as 30 . The experiment is carried out and it is based on number of living node at given instant of time. Below 5\% of residual energy present in the node treated as non-alive node. LE-RPL maintains the full battery up to 70 hours. Then it starts gradually discharge the energy from the battery. After 130 hours of simulation, 25 nodes are alive in the network. The same amount of simulation time, the number of living node compared with different metrics. The X-Axis indicates the simulation time in hours. The Y-Axis indicates number of living nodes. The evaluations made and table-2 shows that number of living node with respect to the simulation time. Fig. 2 shows that number of living node's vs. simulation time. The proposed LE-RPL has improved the network lifetime.
Table 1. Simulation configuration for experiments

\begin{tabular}{|l|l|}
\hline \multicolumn{1}{|c|}{ Parameter } & \multicolumn{1}{c|}{ Value } \\
\hline $\begin{array}{l}\text { Operating } \\
\text { System }\end{array}$ & Contiki 2.7 \\
\hline Node Type & Tmote sky \\
\hline $\begin{array}{l}\text { Minimum DIO } \\
\text { interval }\end{array}$ & 12 \\
\hline Routing Protocol & RPL \\
\hline $\begin{array}{l}\text { MAC/Adaptation } \\
\text { Layer }\end{array}$ & Contiki MAC/6LowPAN \\
\hline $\begin{array}{l}\text { Radio } \\
\text { Environment }\end{array}$ & Unit Disk Graph Medium \\
\hline $\begin{array}{l}\text { Number } \\
\text { Nodes }\end{array}$ & 30 \\
\hline $\begin{array}{l}\text { Simulation } \\
\text { Duration }\end{array}$ & $24 \mathrm{Hrs}$ \\
\hline Full Battery & $3000 \mathrm{~mJ}$ \\
$\begin{array}{l}\text { Transmission } \\
\text { Range }\end{array}$ & $400 \times 400 \mathrm{~m}^{2}$ \\
\hline $\begin{array}{l}\text { Data Packet } \\
\text { Timer }\end{array}$ & $60 \mathrm{sec}$ \\
\hline RPL Parameter & MinHopRankIncrease $=256$ \\
\hline
\end{tabular}

Table 2. Number of living node Vs Simulation time

\begin{tabular}{|r|r|r|r|r|r|r|}
\hline & \multicolumn{6}{|c|}{ Number of living node } \\
\hline $\begin{array}{c}\text { Simul } \\
\text { ation } \\
\text { Time } \\
\text { (h) }\end{array}$ & $\begin{array}{c}\text { Hop } \\
\text { count }\end{array}$ & $\begin{array}{c}\text { E } \\
\text { T }\end{array}$ & RER & $\begin{array}{c}\text { RER } \\
\text { (BDI) }\end{array}$ & Load & $\begin{array}{c}\text { LE- } \\
\text { RPL }\end{array}$ \\
\hline 0 & 30 & 30 & 30 & 30 & 30 & 30 \\
\hline 10 & 30 & 30 & 30 & 30 & 30 & 30 \\
\hline 20 & 30 & 30 & 30 & 30 & 30 & 30 \\
\hline 30 & 30 & 30 & 30 & 30 & 30 & 30 \\
\hline 40 & 26 & 30 & 30 & 30 & 30 & 30 \\
\hline 50 & 23 & 28 & 30 & 30 & 30 & 30 \\
\hline 60 & 19 & 26 & 28 & 30 & 30 & 30 \\
\hline 70 & 18 & 25 & 27 & 30 & 29 & 30 \\
\hline 80 & 19 & 23 & 26 & 29 & 29 & 28 \\
\hline 90 & 17 & 18 & 24 & 27 & 27 & 28 \\
\hline 100 & 13 & 16 & 23 & 27 & 27 & 28 \\
\hline 110 & 10 & 14 & 22 & 26 & 25 & 27 \\
\hline 120 & 0 & 10 & 21 & 26 & 25 & 26 \\
\hline 130 & 0 & 8 & 18 & 22 & 23 & 25 \\
\hline & & & & & & \\
\hline
\end{tabular}




\subsection{Packet delivery ratio}

Packet Delivery Ratio denotes that number of packets received successfully at destination from number of packets send by sender. Or otherwise the ratio of data packets successfully received to the total sent.

$\mathrm{X}$ Axis indicates transmission speed $(\mathrm{RX})$ and $\mathrm{Y}$ Axis indicates Packet delivery ratio [26]. The transmit ratio(TX) is $100 \%$ and receive ratio (RX) is different values are given and tested. As a result, LE-RPL performed well in higher RX value. Hence, packet loss ratio is low in higher $\mathrm{RX}$ value. The proposed metric has minimized the packet loss ratio in all kind of RX value. LE-RPL average packet delivery ratio is above $90 \%$ from the RX value 80 to 100 . Table- 3 shows that the packet delivery ratio in different RX ratio. Fig.3 shows that proposed work (LE-RPL) Packet deliery ratio represented in below.

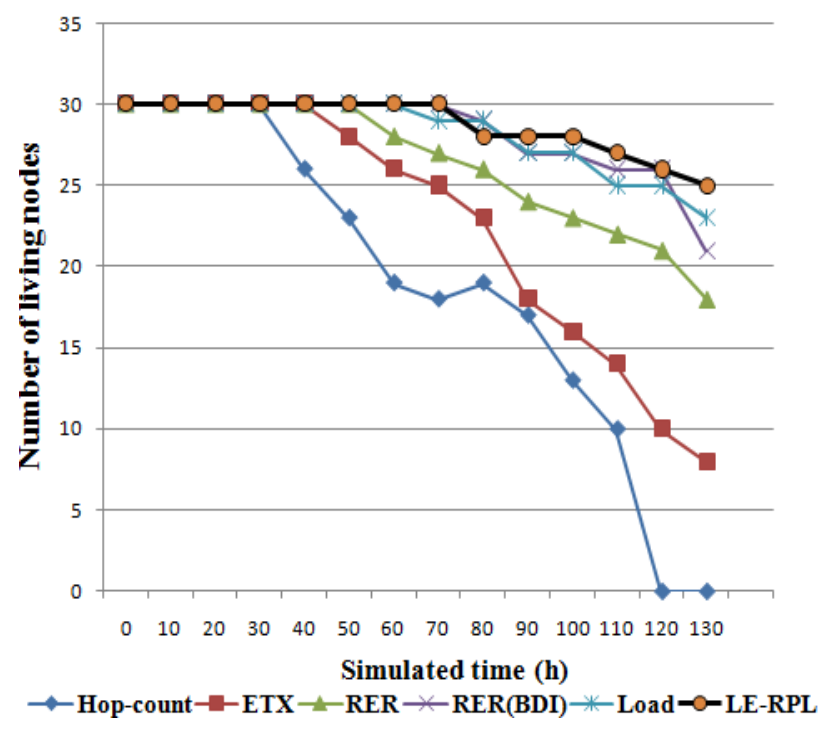

Figure. 2 Number of living nodes vs. simulated time (h)

Table 3. Packet delivery ratio vs. RX ratio

\begin{tabular}{|c|c|c|c|c|c|}
\hline & \multicolumn{5}{|c|}{ PDR (\%) } \\
\hline $\begin{array}{c}\text { RX } \\
\text { Value }\end{array}$ & ETX & RER(BDI) & $\begin{array}{c}\text { Hop- } \\
\text { count }\end{array}$ & Load & $\begin{array}{c}\text { LE- } \\
\text { RPL }\end{array}$ \\
\hline 40 & 55 & 60 & 64 & 66 & 68 \\
\hline 50 & 58 & 63 & 65 & 67 & 70 \\
\hline 60 & 68 & 72 & 76 & 78 & 81 \\
\hline 70 & 77 & 82 & 82 & 84 & 85 \\
\hline 80 & 84 & 87 & 88 & 90 & 90 \\
\hline 90 & 88 & 89 & 90 & 90 & 91 \\
\hline 100 & 88 & 89 & 90 & 92 & 93 \\
\hline
\end{tabular}

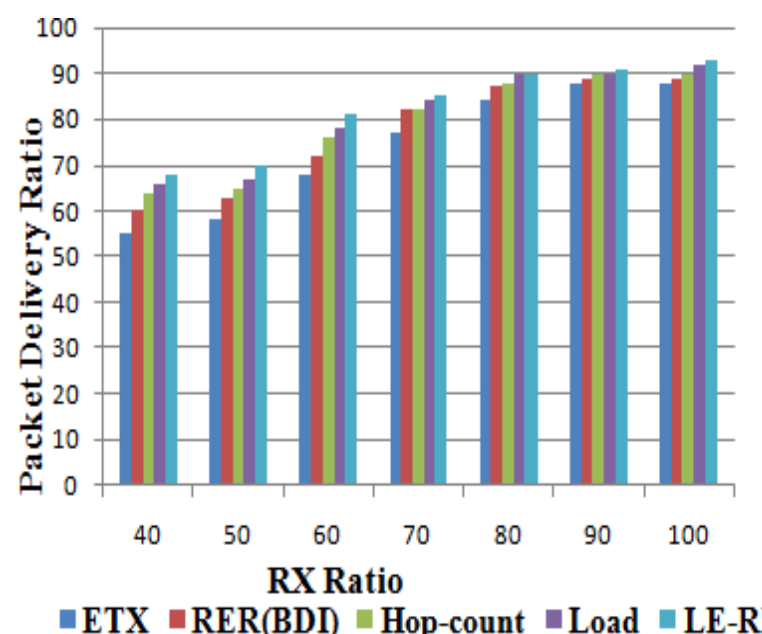

Figure. 3 Packet delivery ratio vs. RX ratio

\section{Conclusion}

Load and BDI based composite routing metric (LE-RPL) is proposed in this paper. In RPL, participant node wants to send the data to DODAG root through parent node. The participant node chooses the best parent based on rank, among the preferred parent in DODAG. The rank value calculates from composite routing metric. The performance of proposed work assessed by the means of Packet delivery ratio (PDR) and network lifetime. The experimental results show that the proposed system provides better performance with less packet loss ratio and improves the network lifetime than existing objective function Hop count, ETX, RER, RER (BDI) and Load. Hence, the proposed work achieves the improved network lifetime ( 25 nodes are alive in after 130 hours of simulation) and packet delivery ratio is keeps on increasing with respect to the RX value. Future work will include the link metric like received signal strength indicator (RSSI) and also planned to use fuzzy parameter based routing decision making in LLN.

\section{References}

[1] A. Al-Fuqaha, M. Guizani, M. Mohammadi, M. Aledhari, and M. Ayyash, "Internet of things: A survey on enabling technologies, protocols, and applications", IEEE Communications Surveys \& Tutorials, Vol.18, No.4, pp. 2347-2376, 2015.

[2] S. Sankar and P. Srinivasan, "Internet Of Things (Iot): A Survey On Empowering Technologies, Research Opportunities And Applications", International Journal of Pharmacy and Technology, Vol.8, No.4, pp.26117-26141, 2016. 
[3] M.N. Moghadam, H.Taheri, and M. Karrari, "Minimum cost load balanced multipath routing protocol for low power and lossy networks", Wireless networks, Vol. 20, No.8, pp. 2469-2479, 2014.

[4] M.N. Moghadam, H. Taheri, and M. Karrari, "Multi-class multipath routing protocol for low power wireless networks with heuristic optimal load distribution", Wireless Personal Communications, Vol.82, No.2, PP.861881,2015 .

[5] A. Hassan, S. Alshomrani, A. Altalhi, and S. Ahsan, "Improved routing metrics for energy constrained interconnected devices in lowpower and lossy networks", Journal of Communications and Networks, Vol.18, No.3, pp. 327-332, 2016.

[6] J. Tripathi, J.C. de Oliveira, and J.P. Vasseur, "A performance evaluation study of rpl: Routing protocol for low power and lossy networks", In: Proc. of International Conf. on Information Sciences and Systems, pp.1-6, 2010. 2010.

[7] N.R. Mohamed, A. Jamil, L.H. Audah, J. Abdullah, and R. Alias, "Performance of NonUniform Duty-Cycled ContikiMAC in Wireless Sensor Networks", International Journal of Electrical and Computer Engineering, Vol.7, No.2, pp.942-949, 2017.

[8] P.K. Reddy and R.Babu, "An Evolutionary Secure Energy Efficient Routing Protocol in Internet of Things", International Journal of Intelligent Engineering and Systems, Vol.10, No.3, pp.337-346, 2017.

[9] O.Gaddour, and K. Anis, "RPL in a nutshell: A survey", Computer Networks, Vol.56, No.14, pp. 3163-3178, 2012.

[10] O. Iova, P.Pietro, I. Timofei, and K. Casba, "RPL: The Routing Standard for the Internet of Things... Or Is It?.", IEEE Communications Magazine, Vol.54, No.12, pp.16-22,2016.

[11] P. Karkazis, T. Panagiotis, C.L. Helen, S. Lambros, P. Ioannis, and Z. Theodore, "Evaluating routing metric composition approaches for QoS differentiation in low power and lossy networks", Wireless networks, Vol.10, No.6, pp.1269-1284,2013.

[12] B.Y. Muneer, A.B. Firas, and O.A. Odeh," Energy-Aware Objective Function for Routing Protocol in Internet of Things", International Journal on Communications Antenna and Propagation, Vol.7, No.3, 2017.

[13] G. Xu and L. Gang, "Multipath routing protocol for DAG-based WSNs with mobile sinks", In: Proc. of International Conf. on Computer
Science and Electronics Engineering, pp.16781682, 2013.

[14] O. Iova, T. Fabrice, and N. Thomas, "Using multiparent routing in RPL to increase the stability and the lifetime of the network", $A d$ Hoc Networks, Vol.29, No.6, pp.245-262, 2015.

[15] P. Karkazis, H.C. Leligou, L. Sarakis T. Zahariadis, P. Trakadas, T.H. Velivassaki, and C. Capsalis, "Design of primary and composite routing metrics for RPL-compliant Wireless Sensor Networks", In: Proc. of International Conf. on Telecommunications and Multimedia, pp.13-18, 2012.

[16] D.S. De Couto , D. Aguayo, J. Bicket, and R. Morris, "A high-throughput path metric for multi-hop wireless routing", Wireless Networks, Vol.11, No.4,pp.419-434,2005.

[17] M. Qasem, A.Al-Dubai, I.Romdhani, B.Ghaleb, and W.Gharibi, "A new efficient objective function for routing in Internet of Things paradigm", In: Proc. of International conf. on Standards for Communications and Networking, pp.1-7, 2016.

[18] J.P. Yunis and D. Diego, "Energy efficient routing performance evaluation for LLNs using combined metrics", In: Proc. of Biennial Congress of Argentina, pp.741-746, 2014.

[19] L. Nguyen, L. Lefevre, and D. GenonCatalot,"A composite metric for dynamic routing in networked control systems", In: Proc. of International Conf. on Industrial Informatics, pp.672-675, 2016.

[20] P.O. Kamgueu, N. Emmanuel, and N.D. Thomas, "On design and deployment of fuzzybased metric for routing in low-power and lossy networks", In: Proc. of International conf. on Local Computer Networks Conference Works, pp.789-795, 2015.

[21] O.Gaddour, K.Anis, B.Nauha, and A.Mohamed, "OF-FL: QoS-aware fuzzy logic objective function for the RPL routing protocol",In: Proc. International Symposium on Modelling and Optimization in Mobile, Ad Hoc, and Wireless Networks, pp.365-372, 2014.

[22] S. Capone, B. Riccardo, A.Nicola, S.Deminico, and B.Gennaro, "An energy efficient and reliable composite metric for RPL organized networks", In: Proc. of International Conf. on Embedded and Ubiquitous Computing, pp.178184, 2014.

[23] F. Osterlind, A. Dunkels, J. Eriksson, N. Finne, and T. Voigt," Cross-level sensor network simulation with cooja", In: Proc of International Conference on Local computer networks, proceedings, pp.641-648, 2006. 
[24] M.F. Youssef, M.F. K.M.F. Elsayed, and A.H. Zahran, "Adaptive radio duty cycling in ContikiMAC: Proposal and analysis", In: Proc. Of World Forum on Internet of Things, pp.491495, 2014.

[25] S. Yang, F. Dai, M. Cardei, J. Wu, and F. Patterson," On connected multiple point coverage in wireless sensor networks." International Journal of Wireless Information Networks, Vol.13, No.4, pp.289-301, 2006.

[26]E.G. Davis, C. Anna, and D. Ilker, "Improving packet delivery performance of publish/subscribe protocols in wireless sensor networks," Sensors, Vol.13, No.1, pp.648-680, 2013. 